\title{
IDENTIFICATION OF CLASSES OF BILINEAR TIME SERIES MODELS
}

\author{
ANTHONY E. USORO \\ Department of Mathematics and Statistics \\ Akwa Ibom State University \\ Mkpat Enin, Akwa Ibom State \\ Nigeria \\ e-mail: toskila2@yahoo.com
}

\begin{abstract}
In this paper, two classes of bilinear time series model are obtained under certain conditions from the general bilinear autoregressive moving average model. Bilinear autoregressive (BAR) and bilinear moving average (BMA) models have been identified. From the general bilinear model, BAR and BMA models have been proved to exist for $q=Q=0,=>j=0$, and $p=P=0,=>i=0$, respectively. These models are found useful in modelling most of the economic and financial data.
\end{abstract}

\section{Introduction}

Bilinear time series model is a class of time series model, which assume both linear and non-linear components of autoregressive moving average processes. The linear part of the bilinear model is the aggregation of autoregressive and moving average processes, while the non-linear part is the product of the two processes. The general bilinear

2010 Mathematics Subject Classification: 37M10.

Keywords and phrases: autoregressive model, moving average model, bilinear autoregressive model, bilinear moving average model.

Received April 14, 2017

(C) 2017 Scientific Advances Publishers 
time series model as defined by Kendell and Ord [5]; Bibi and Oyet [1], and Iwueze [4] is

$$
X_{t}=\sum_{i=1}^{p} \phi_{i} X_{t-i}+\sum_{j=1}^{q} \theta_{j} \epsilon_{t-j}+\sum_{i=1}^{P} \sum_{j=1}^{Q} \beta_{i j} X_{t-i} \epsilon_{t-j}+\epsilon_{t}
$$

where $X_{t}$ is the bilinear process, $X_{t-i}$ and $\epsilon_{t-j}$ are time varying autoregressive and moving average processes, respectively, with $\phi_{i}$ and $\theta_{j}$ as their respective parameters; $\beta_{i j}$ are the non-linear parameters of $X_{t-i} \epsilon_{t-j}, 1 \leq i \leq p, 1 \leq j \leq q$ and $1 \leq i \leq P, 1 \leq j \leq Q ; \epsilon_{t}$ is the error term, $\epsilon_{t} \sim N\left(0, \sigma_{\epsilon_{t}}^{2}\right)$. Model (1) is a difference equation for a time varying bilinear process of order $(p, q, P, Q)$, also known as $B L(p, q, P, Q)$.

Granger and Anderson [3] identified a complete non-linear part of bilinear model with $p=q=0$. That is,

$$
X_{t}=\sum_{i=1}^{P} \sum_{j=1}^{Q} \beta_{i j} X_{t-i} \epsilon_{t-j}+\epsilon_{t}
$$

It is expressed in the matrix form as

$$
X_{t}=\left(X_{t-1}, X_{t-2}, \ldots, X_{t-P}\right)^{\mathrm{l}}\left(\epsilon_{t-1}, \epsilon_{t-2}, \ldots, \epsilon_{t-Q}\right) \underline{\beta}+\epsilon_{t},
$$

where $\beta$ is the matrix of parameters of order $P$ and $Q$.

$$
\underline{\beta}=\left(\beta_{i j}\right), i=1,2, \ldots, P ; j=1,2, \ldots, Q .
$$

Usoro and Omekara [7] used the non-linear part of bilinear time series model to express pure diagonal bilinear model. From model (2), if $i=j$, the coefficient matrix $\underline{\beta}=\left(\beta_{i j}\right), i=1,2, \ldots, P ; j=1,2, \ldots, Q$ of the nonlinear part of the bilinear model exists with elements in the principal diagonal of the matrix. Maravell [6] and Franses [2] ascertained the reliability of bilinear model and its performance over alternative linear models when modelling economic and financial data. 


\section{Model Identification}

This section deals with identification of classes of bilinear models from model (1) under certain conditions.

\subsection{Bilinear autoregressive (BAR) model}

Given the difference equation

$$
X_{t}=\sum_{i=1}^{p} \phi_{i} X_{t-i}+\sum_{j=1}^{q} \theta_{j} \epsilon_{t-j}+\sum_{i=1}^{P} \sum_{j=1}^{Q} \beta_{i j} X_{t-i} \epsilon_{t-j}+\epsilon_{t} .
$$

If $q=Q=0, \Rightarrow j=0$, the model becomes

$$
X_{t}=\sum_{i=1}^{p} \phi_{i} X_{t-i}+\sum_{i=1}^{P} \beta_{i 0} X_{t-i} \epsilon_{t}+\epsilon_{t}
$$

where $\beta_{i 0}$ are the coefficients of the non-linear autoregressive part of the bilinear model. $\phi_{i}, X_{t-i}, \epsilon_{t}$ are as described earlier.

Proof. Let $X_{t}=Y_{t}+Z_{t}+\epsilon_{t}$, where,

$$
Y_{t}=\sum_{i=1}^{p} \phi_{i} X_{t-i}+\sum_{j=1}^{q} \theta_{j} \epsilon_{t-j}
$$

and

$$
Z_{t}=\sum_{i=1}^{P} \sum_{j=1}^{Q} \beta_{i j} X_{t-i} \epsilon_{t-j}
$$

From Equation (5), if $q=0,=>j=0$, the model becomes

$$
Y_{t}=\sum_{i=1}^{p} \phi_{i} X_{t-i}
$$

Equation (6) is expressed in matrix form as

$$
Z_{t}=\left(X_{t-1}, X_{t-2}, \ldots, X_{t-P}\right)^{\mathrm{l}}\left(\epsilon_{t-1}, \epsilon_{t-2}, \ldots, \epsilon_{t-Q}\right) \underline{\beta} .
$$

The matrix expansion is 


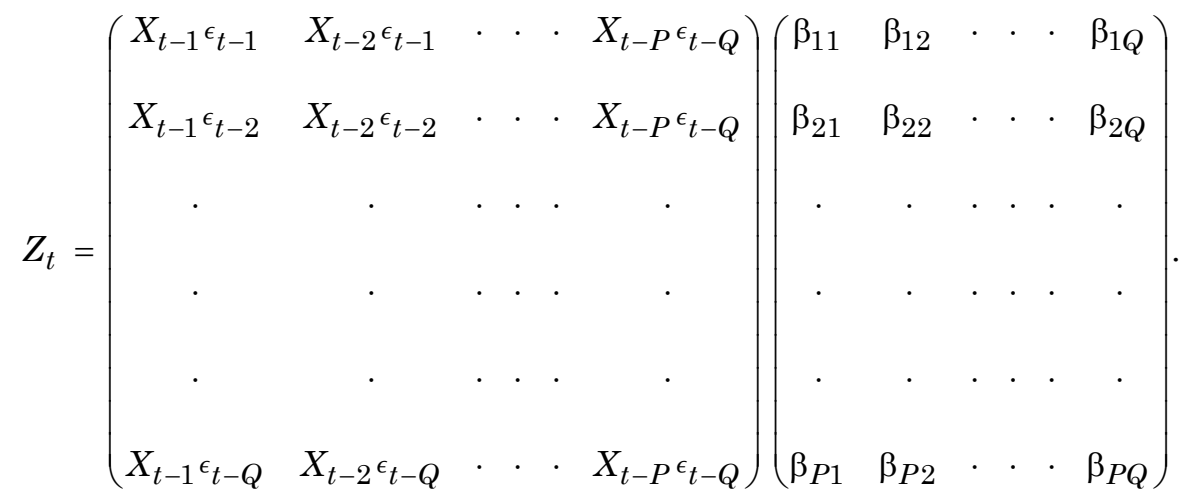

From (8), if $Q=0,=>j=0$, then

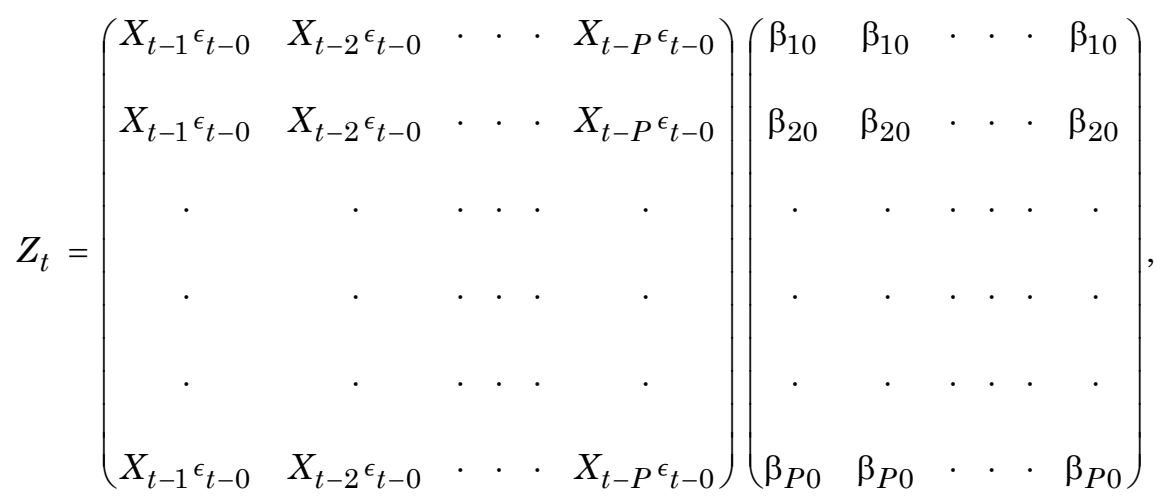

$$
Z_{t}=\left(\begin{array}{ll}
X_{t-1} \epsilon_{t-0} X_{t-2}{ }^{\epsilon} t-0 \\
\cdots X_{t-P} \epsilon_{t-0}
\end{array}\right)\left(\begin{array}{c}
\beta_{10} \\
\beta_{20} \\
\cdot \\
\cdot \\
\cdot \\
\beta_{P 0}
\end{array}\right)=\beta_{10} X_{t-1} \epsilon_{t}+\beta_{20} X_{t-2} \epsilon_{t}
$$




$$
Z_{t}=\sum_{i=1}^{P} \beta_{i 0} X_{t-i} \epsilon_{t}
$$

Combining (7) and (10), $X_{t}$ becomes,

$$
X_{t}=\sum_{i=1}^{p} \phi_{i} X_{t-i}+\sum_{i=1}^{P} \beta_{i 0} X_{t-i} \epsilon_{t}+\epsilon_{t}
$$

This completes the proof. Hence, Equation (4) is a bilinear autoregressive (BAR) model of order $p, P$. That is $B L(p, P)$.

\subsection{Bilinear moving average (BMA) model}

Corollary. Given the difference equation

$$
X_{t}=\sum_{i=1}^{p} \phi_{i} X_{t-i}+\sum_{j=1}^{q} \theta_{j} \epsilon_{t-j}+\sum_{i=1}^{P} \sum_{j=1}^{Q} \beta_{i j} X_{t-i} \epsilon_{t-j}+\epsilon_{t} .
$$

If $p=P=0,=>i=0$, the model becomes

$$
X_{t}=\sum_{j=1}^{q} \theta_{j} \epsilon_{t-j}+\sum_{j=1}^{Q} \beta_{0 j} X_{t} \epsilon_{t-j}+\epsilon_{t}
$$

where $\beta_{0 j}$ are the coefficients of the non-linear moving average part of the bilinear model. $\theta_{j}, X_{t}, \epsilon_{t-j}$ are as described earlier.

Proof. Let $X_{t}=R_{t}+S_{t}+\epsilon_{t}$, where

$$
R_{t}=\sum_{i=1}^{p} \phi_{i} X_{t-i}+\sum_{j=1}^{q} \theta_{j} \epsilon_{t-j}
$$

and

$$
S_{t}=\sum_{i=1}^{P} \sum_{j=1}^{Q} \beta_{i j} X_{t-i} \epsilon_{t-j}
$$

From Equation (12), if $p=0,=>i=0$, the model becomes 


$$
R_{t}=\sum_{j=1}^{q} \theta_{j} \epsilon_{t-j}
$$

Equation (13) is expressed in matrix form as

$$
S_{t}=\left(X_{t-1}, X_{t-2}, \ldots, X_{t-P}\right)^{\mathrm{I}}\left(\epsilon_{t-1}, \epsilon_{t-2}, \ldots, \epsilon_{t-Q}\right) \underline{\beta} .
$$

The matrix expansion is

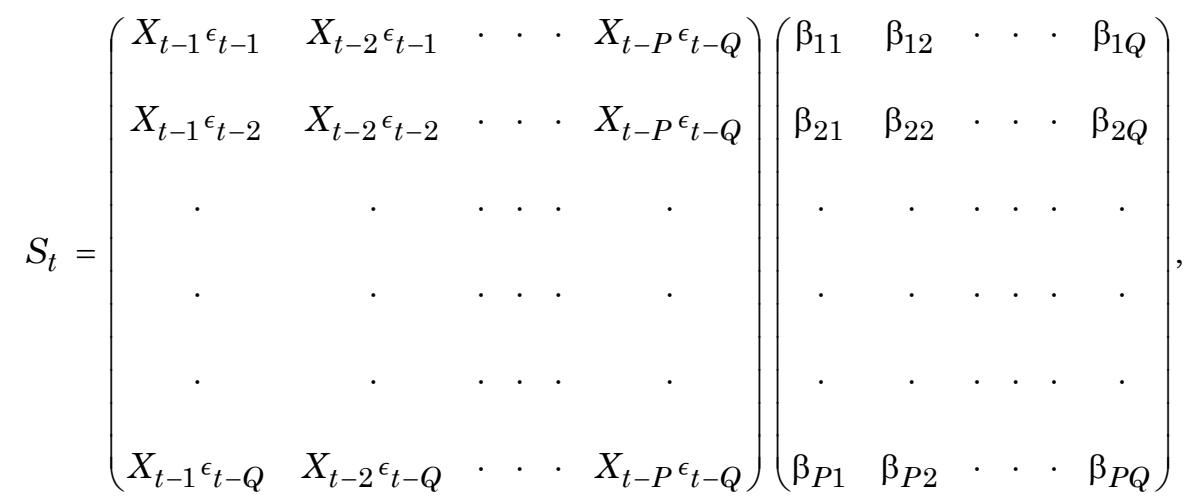

From (8), if $p=0,=>i=0$, then

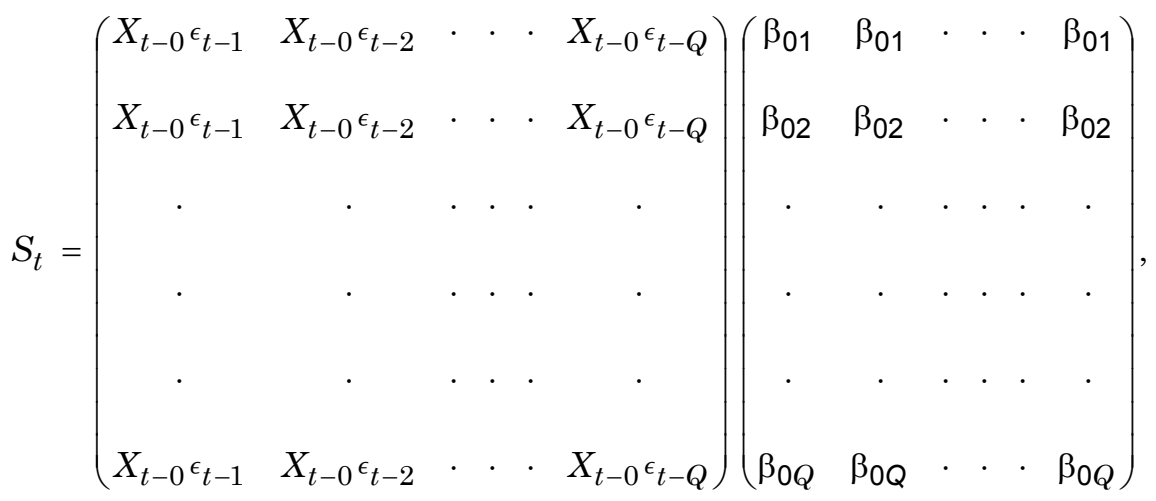




$$
S_{t}=\left(X_{t-0} \epsilon_{t-1} X_{t-0} \epsilon_{t-2} \cdots X_{t-0} \epsilon_{t-Q}\right)\left(\begin{array}{c}
\beta_{01} \\
\beta_{02} \\
\cdot \\
\cdot \\
\cdot \\
\beta_{0 Q}
\end{array}\right)=\beta_{01} X_{t} \epsilon_{t-1}+\beta_{02} X_{t} \epsilon_{t-2}
$$

Combining (14) and (17), $X_{t}$ becomes,

$$
X_{t}=\sum_{j=1}^{q} \theta_{j} \epsilon_{t-j}+\sum_{j=1}^{Q} \beta_{0 j} X_{t} \epsilon_{t-j}+\epsilon_{t}
$$

This completes the proof. Hence, Equation (11) is a bilinear moving average (BMA) model of order $q, Q$. That is $B L(q, Q)$.

\section{Conclusion}

Bilinear time series model is a class of time series model that has found applications in various areas of time series modelling, especially when dealing with data series that assume non-linearity behaviour. Bilinear time series model, like ordinary autoregressive moving average model, is characterised by individual autoregressive and moving average processes. The introduction of the non-linear part is what distinguished a bilinear process from the linear process. Here, bilinear autoregressive and bilinear moving average models have been established and proved. 


\section{References}

[1] A. Bibi and A. J. Oyet, Estimation of some bilinear time series models with time varying coefficients, AMS (1991).

[2] P. H. Franses, Time series models for business and economic forecast, Journal of Time Series Annals 16 (1998), 509-529.

[3] C. W. Granger and A. P. Anderson, Introduction to Bilinear Time Series Models, Vandenhoeck and Ruprecht, 1978.

[4] I. S. Iwueze, Vectorial representation and its application to covariance analysis of super-diagonal bilinear time series models, The Physical Scientist 1(1) (2002), 85-96.

[5] M. Kendell and Keith J. Ord, Time Series. Third Edition, Halsted Press, Third Avenue, New York, 1990.

[6] A. Maravall, An application of non-linear time series forecasting, Journal of Business and Economic Statistics 1 (1983), 66-74.

[7] A. E. Usoro and C. O. Omekara, Pure diagonal bilinear moving average vector models, Global Journal of Mathematical Sciences 7(2) (2008), 137-145. 